\title{
Prevalence of Candida in Blood Cultures from in patients at a Teaching Hospital in Brazil
}

\author{
Vilson Sovio Oliveira de Macedo ${ }^{1}$, Sebastião Carlos de Sousa Oliveira ${ }^{2}$, \\ Maria Rosineida Paiva Rodrigues ${ }^{2}$, Camila Gomes Virginio Coelho ${ }^{1}$ and \\ Francisco Cesar Barroso Barbosa ${ }^{1^{*}}$
}

\author{
${ }^{1}$ Postgraduate Program in Health Sciences, Federal Universityof Ceará, \\ Sobral, Ceará, Brazil \\ ${ }^{2}$ Medical School, Federal Universityof Ceará, Sobral, Ceará, Brazil
}

*Corresponding author

\begin{tabular}{|l|}
\hline Ke y w o r d s \\
Candida albicans, \\
Candidemia, \\
Frequence, \\
Nosocomial \\
Infection, Tertiary \\
hospital
\end{tabular}

\section{A B S T R A C T}

Candidemia is a serious public health problem and is associated with high morbidity and mortality rates. This study aimed to determine the prevalence of Candida species isolated from inpatients with candidemia at a teaching hospital in Brazil; characterize patients; correlate the risk factors and investigate the use of antibiotics. Medical records of 54 hospitalized patients from October 2013 to July 2015 were evaluated. The species identification was performed by automated system Vitek ® 2 . In this period there were 19,962 hospitalizations, observing a frequency of 2.7 cases per 1,000 admissions. The prevalent species were Candida albicans (29.6\%), C. tropicalis (29.6\%), and C. parapsilosis (29.6\%). Neonatal sepsis was the clinical condition more related to candidemia. The highest incidence of Candida species was observed in patients undergoing invasive procedures. All subjects studied were submitted to prolonged antibiotic therapy. The greatest diversity of Candida species was isolated from the Intensive Care Unit (ICU) and adult wards. The mortality rate found was $51.0 \%$, of which $78.0 \%$ were ICU patients. These results reinforce the need to follow the clinical course of hospitalized patients for Candida infection through epidemiological surveillance in order to contribute to the action plan

\section{Introduction}

Candida spp. is the most common cause of fungal infections in hospitalized patients. They are also frequent pathogens in blood infections, and are associated with high morbidity and mortality (Yesilkaya et al.,
2017). In the last two decades, there has been an increase in occurrence of infections by species of Candida (Bailly et al., 2016).

This increase has been attributed, in part, to the increase in susceptible patients, such as patients with neoplasia or degenerative 
diseases, HIV seropositives, patients in intensive care units, receiving immunosuppressing therapies associated with transplants and broad-spectrum antibiotics therapies. All of this makes Candida infection a problem in medical practice in hospitals (Ala-Houhala et al., 2019).

The frequent usage of antibiotics, central venous catheters and other invasive devices, abdominal surgery and prolonged stays in Intensive Care Units (ICU) predispose patients to a risk of infection by Candida reflected in lower survival rates to any blood infection (Yesilkaya et al., 2017).

The determining factor for the predominance of some pathology is not simply by the presence of Candida spp. in human microbiota, but changes in the host's defenses that alter the parasite/host balance favoring the invasion of this yeast through various mechanisms, including the translocation of Candida spp. to the mesenteric capillaries (Chatelon et al., 2019). Changes in skin and mucous caused by this yeast, like hydration, $\mathrm{pH}$ e nutrient levels can alter the normal microbiota and lead to infections caused by this fungus. Candida albicans is the main species detected on incidences of candidemias but some studies indicate an increase of candidemia due to other species, such as $C$. glabrata, C. parapsilosis, $C$. tropicalis and $C$. krusei, representing a new challenge to empirical therapy and prevention strategies (Lin et al., 2018; Pinto-Magalhães et al., 2019).

The incidence of infections by Candida spp. have been increasing considerably in the last years, thus health professionals have an important role to play in acquiring knowledge of necessary steps for diagnosis, control and treatment of invasive infections by these pathogens. It is then essential to accomplishment blood cultures for diagnosis of fungemias, as it is an exam of great value for laboratorial diagnosis and infection prevalence (Ezenwa et al., 2017).

Therefore, the aim of this study was to detect the number of cases of blood cultures positive for Candida spp. from inpatients at Santa Casa de Misericordia of Sobral (SCMS), a teaching hospital focused on tertiary care, a reference in the North of the state of Ceará - Brazil; and characterize the patients for associated risk factors, use of invasive devices and prior usage of antibiotics.

\section{Materials and Methods}

A retrospective observational study was carried out from October 2013 to July 2015, to analyze the prevalence of Candida spp. in blood cultures at SCMS, reference hospital for 61 municipalities in the Macro-Region of Sobral, covering approximately 2 million inhabitants, with $92.0 \%$ of its total installed area at the service of the Unified Health System.

Candidemia cases were considered in adult patients or neonates with at least one positive blood culture for any Candida species during a single hospitalization for more than 30 days, or with an invasive device for over 15 days.

The patients analyzed in this study were selected from the analysis of results of blood cultures generated by SCMS's microbiology laboratory. The candidemia patients' clinical and epidemiological data were gathered from revision of medical records and stored in databases using Microsoft Excel 2007 version 9.0.2812. The information of interest obtained from the records were the patients' age, gender, mycological diagnosis, reason for admission, prior exposures to antibiotics, length of hospital stay, invasive procedures performed during the stay and other risk factors for candidemia. 
The present study was conducted according to the Declaration of Helsinki, and the protocol was approved by the Institutional Ethics Committee of the State University of Vale of Acaraú, Sobral, Ceará, Brazil (Protocol n ${ }^{\circ}$ 644,365).

The biological samples were collected at SCMS by hospital professionals from its Laboratory for Clinical Analysis. The samples were immediately transported to the institution's microbiology laboratory, where they were identified.

Phenotypic identification and susceptibility to antimicrobials were determined by the VITEK®2 automated system (BioMérieux, Marcy-l'Etoile, France) at SCMS microbiology laboratory.

\section{Results and Discussion}

For the time period chosen for the study a total of 19,962 were admitted to SCMS, 54 of which presented blood cultures positive for Candida spp., revealing an incidence rate of 2.7 cases per 1,000 inpatients. The yeasts identified were of the following species: $C$. albicans (29.6\%), C. tropicalis (29.6\%), C. parapsilosis (29.6\%), C. famata (5.0\%), C. lusitaniae (1.8\%), and other species. Most of the candidemias were diagnosed in patients aged 0 to 14 years $(44.4 \%)$, the frequency of cases among men being $46.3 \%$ and $53.7 \%$ among women. The most documented reasons for hospitalization prior to candidemia diagnosis were neonatal sepsis (16.6\%), pneumonia (16.6\%) and Traumatic Brain Injury (TBI) (14.8\%) (Table 1).

The frequency of isolation of Candida species varied with gender, patients' age and underlying diseases. C. albicans was isolated with greater frequency in female $(68.7 \%)$, aged 0 to 14 years $(50.0 \%)$, in patients stricken with pneumonia (44.4\%) and with
TBI $(37.5 \%)$. On the other hand, for patients diagnosed with neonatal sepsis, $C$. parapsilosis was detected in $55.5 \%$ of candidemia cases and $C$. tropicalis was isolated in $80.0 \%$ of patients with respiratory disorders. All patients studied had been submitted to antibiotics therapy prior to candidemia diagnosis (Table 1).

The distribution of $C$. albicans and nonalbicans Candida by different risk factors associated with the patients is presented in table 2. Among the observed patients, 24 presented some risk factors, particularly anemia and trauma for all fungal species found, with incidences of $C$. tropicalis (37.5\%), C. parapsilosis $(37.5 \%)$ and $C$. albicans $(12.5 \%)$ in patients with anemia and C. tropicalis $(33.3 \%)$, C. parapsilosis $(33.3 \%)$ and $C$. albicans $(16.6 \%)$ in patients with trauma. However, the greater incidence of $C$. albicans $(60.0 \%)$ was detected in prematurity (Table 2).

C. albicans was the most isolated species of patients with candidemia undergoing invasive procedures. Among these procedures orotracheal intubation, nasogastric tube and tracheostomy had higher prevalence of isolated strains, as indicated in table 3.

Among the data obtained from exposure prior to antibiotic treatment in patients with candidemia, of the 17 distinct antibiotics used, the most frequent were vancomycin $(17.1 \%)$, meropenem (11.4\%), oxacillin (9.2\%) cefepime $(8.5 \%)$, and imipenem $(7.8 \%)$. In relation to Candida spp. more prevalent in candidemias after antibiotic therapy, $C$. albicans was isolated in $50.0 \%, 45.4 \%$, $41.6 \%$, and $38.4 \%$ of cases of candidemia after the use of meropenem, imipenem, vancomycin, and oxacillin, respectively. While C. tropicalis occurred in $50.0 \%$ of candidemias after the use of cefepime (Table 4). 
In graph 1, we may observe the distribution of species of Candida by hospital sector. It shows a high percentage of $C$. albicans isolation in all hospital sectors except in the nursery. In general, the highest diversity of Candida spp. was isolated in the adult ICU and the infirmary.

The mortality rate observed was of $51.8 \%$, of which $78.5 \%$ were adult ICU, neonatalor pediatric patients $(42.8 \%, 14.3 \%$ and $21.4 \%$, respectively). Among these patients the most common species was C. tropicalisat $46.4 \%$, and $53.5 \%$ of them were treated with antifungal agents (fluconazole or amphotericin B). The reasons for lack of treatment for some patients were hospital transfers, death before a final diagnostic and for some there was no documented reason in their records. A direct correlation between candidemia and the cause of death of these patients can't be drawn due to multiple associated pathologies (Table 5).

Some studies have shown that incidences of candidemia have been on the rise for many hospitals throughout the world (Colombo et al., 2013; Vibhor et al., 2014; Israel et al., 2019), but in some metropolitan regions of the USA the number of cases is decreasing (Cleveland et al., 2015). In our research, we encountered a rate of 2.7 cases per 1,000 hospitalizations, similar to what is reported by studies conducted in other regions of Brazil (Motta et al., 2010; Colombo et al., 2013; Wille et al., 2013). Incidences of candidemia have been reported in some countries like Denmark (0.41 cases per 1,000 hospital admissions) (Arendrup et al., 2011), Israel (0.50 cases per 1,000 admissions) (Rennert et al., 2000), China (0.53 cases per 1,000 admissions) (Li et al., 2013), India (7.76 cases per 1,000 admissions) (Vibhor et al., 2014), and USA (0.14 cases per 1,000 admissions) (Cleveland et al., 2015). In Brazil, studies conducted in public hospital centers from the South and Southeast regions and from Federal
District indicate rates between 1.2 and 2.4 cases per 1,000 hospital admissions (Motta et al., 2010; Colombo et al., 2013; Wille et al., 2013) and in the Northeast region a higher rate of 3.9 cases per 1,000 admissions (Hinrichsen et al., 2008). The different rates between the aforementioned countries may reflect demographic and socioeconomic differences, variations in healthcare practices, different diagnosis and different prolonged antibiotic usage patterns (Epelbaum and Chasan, 2017).

Newborns and children have historically been populations with some of the highest rates of candidemia (Shetty et al., 2005; Chang et al., 2008; Roilides, 2011; Ezenwa et al., 2017) similar to what was observed by this study, in which children under 14 years old represented $44.4 \%$ of patients stricken with candidemia.

In this research $C$. albicans, $C$. tropicalis and $C$. parapsilosis were predominant and the three yeast species had the same frequency (29.6\%). For most of the world, C. albicans accounts for $40 \%$ to $70 \%$ of cases of candidemia (Bassetti et al., 2006; Horn et al., 2009; Dutta and Palazzi, 2011; Al-Rawahi and Roscoe, 2013). However, this distribution has been changing over the last decade, with reports of an ever-increasing percentage of C.non-albicans cases, probably due to changes in antifungal therapy and the increase in the usage of azoles for prophylaxis or empirical treatment (Lortholary et al., 2011; Epelbaum and Chasan, 2017). This distribution of species varies according to the countries, thus $C$. glabrata and $C$. albicans were the most commonly isolated species in Portugal and Canada, whereas $C$. albicans and $C$. parapsilosis were the most common in Spain (St-Germainet al., 2008; Labbé et al., 2009; Ortega et al., 2011). This variation is probably the result of reductions in susceptibility and the emergence of resistant strains. In general, however, the three most prevalent species of Candida are $C$. albicans, $C$. tropicalis and $C$. 
parapsilosis (Al-Rawahi and Roscoe, 2013) as was also observed by our study.

Most cases of candidemia are believed to be endogenously acquired through translocation of Candida into the gastrointestinal tract (Allert et al., 2018). However, haematogenous infections by Candida spp. can also occur exogenously, through contact with health professionals who have patients with central catheters, implants of contaminated prosthesis, as well as through parenteral administration of contaminated solutions (Epelbaum and Chasan, 2017).

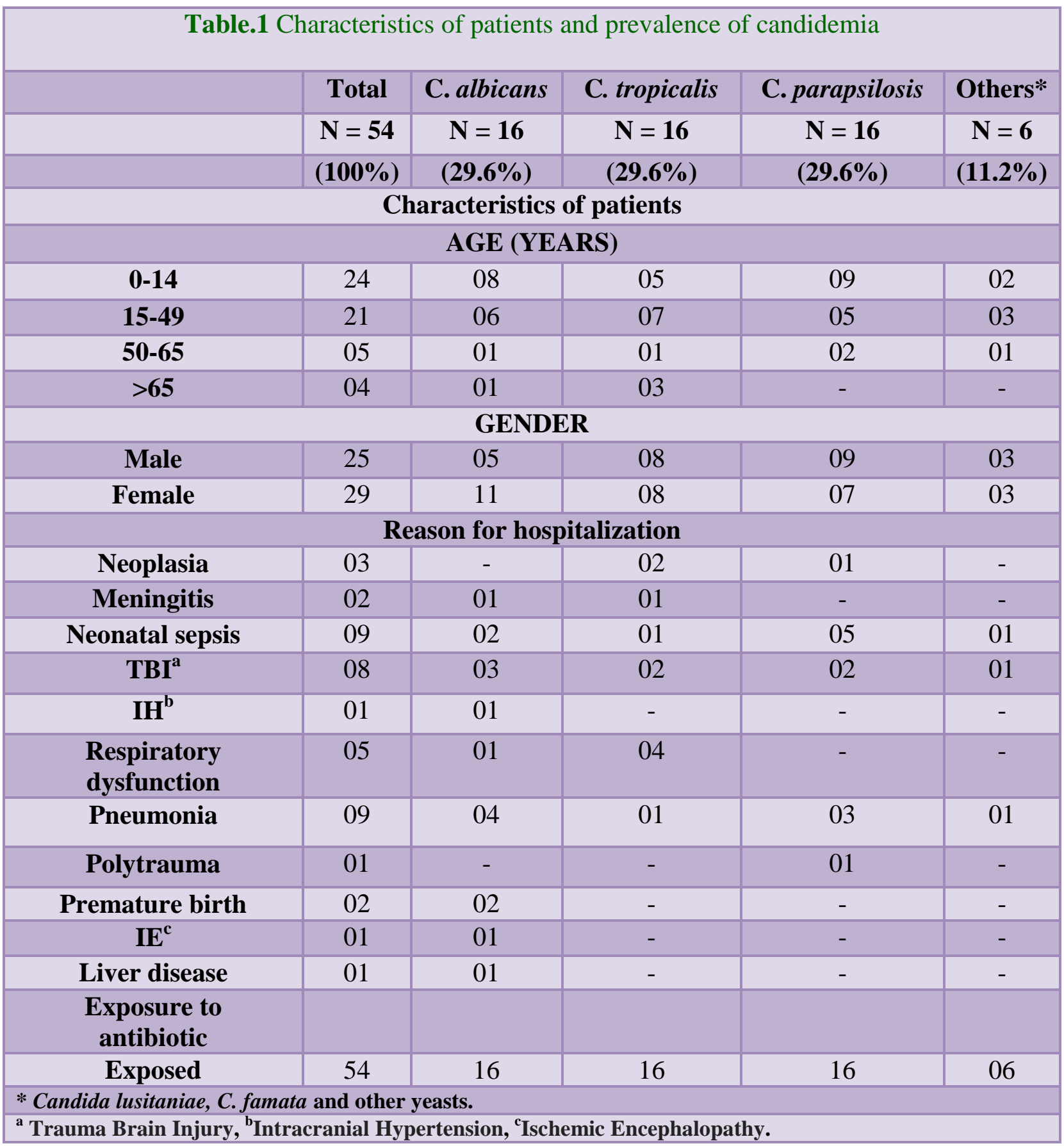




\begin{tabular}{|c|c|c|c|c|c|}
\hline \multicolumn{7}{|c|}{ Table.2 Risk factors in patients with candidemia } \\
\hline Riskfactors & C. albicans & C. tropicalis & C. parapsilosis & Others* & Total \\
\hline Diabetes & 01 & - & 01 & - & 02 \\
\hline Pneumonitis & - & 01 & - & - & 01 \\
\hline Trauma & 01 & 02 & 02 & 01 & 06 \\
\hline TransplantPregnant & 01 & - & - & 01 & 02 \\
\hline Prematurity & 03 & - & 01 & 01 & 05 \\
\hline AnemiaImmunosuppression & 01 & 03 & 03 & 01 & 08 \\
\hline Total & 07 & 06 & 07 & 04 & 24 \\
\hline *Candida lusitaniae, C. famata and other yeasts. & & & & \\
\hline
\end{tabular}

\begin{tabular}{|c|c|c|c|c|c|}
\hline \multicolumn{6}{|c|}{ Table.3 Invasive procedures performed in patients with candidemia } \\
\hline Invasive procedures & C. albicans & C. tropicalis & C. parapsilosis & Others* & Total \\
\hline Central catheter & 03 & 03 & 05 & 02 & 13 \\
\hline Dialysis catheter & 02 & 03 & 02 & - & 07 \\
\hline Urinary catheter & 03 & 03 & 03 & 01 & 10 \\
\hline Nasogastric tube & 07 & 06 & 06 & 01 & 20 \\
\hline Thoracic drainage & 01 & - & - & - & 01 \\
\hline Lumbar puncture & 01 & 01 & 01 & 01 & 04 \\
\hline Endotracheal intubation & 08 & 03 & 04 & 01 & 16 \\
\hline Tracheostomy & 04 & - & 02 & 01 & 07 \\
\hline Total & 29 & 19 & 23 & 07 & 78 \\
\hline
\end{tabular}

\begin{tabular}{|c|c|c|c|c|c|}
\hline \multicolumn{5}{|c|}{ Table.4 Prior exposure to antibiotics and Candida species isolated } \\
\hline Antibiotics & C. albicans & C. tropicalis & C. parapsilosis & Others* & Total \\
\hline Cefepime & 01 & 06 & 04 & 01 & 12 \\
\hline Imipenem & 05 & 02 & 03 & 01 & 11 \\
\hline Ceftriaxone & 03 & 03 & 02 & - & 08 \\
\hline Polymyxin & 02 & 01 & 02 & 01 & 06 \\
\hline Vancomycin & 10 & 04 & 08 & 02 & 24 \\
\hline Meropenem & 08 & 03 & 04 & 01 & 16 \\
\hline Oxacillin & 05 & 04 & 02 & 02 & 13 \\
\hline Penicillin & 04 & 01 & 03 & 01 & 09 \\
\hline Gentamicin & 04 & - & 03 & 01 & 08 \\
\hline Clindamycin & 01 & 02 & 04 & 01 & 08 \\
\hline Others** & 08 & 06 & 09 & 02 & 25 \\
\hline Total & 51 & 32 & 44 & 13 & 140 \\
\hline * Candida lusitaniae, C. famata and other yeasts. & & & \\
\hline **Tazocin, Ampicillin, Cephalothin, Metronidazole, Clindamycin, Teicoplanin, Cefotaxime, andCiprofloxacin. \\
\hline
\end{tabular}




\begin{tabular}{|c|c|c|}
\hline \multicolumn{3}{|c|}{ Table.5 Factors associated with mortality in candidemia patients } \\
\hline & After 30 days of hospitalization \\
\hline Variable & Survived $(\mathrm{n}=26)$ & Death $(\mathrm{n}=28)$ \\
\hline Gender (male:female) & $02: 04$ & $05: 03$ \\
\hline Adult ICU & 06 & 12 \\
\hline Neonatal ICU & 09 & 04 \\
\hline Pediatric ICU & 04 & 05 \\
\hline Infirmary & 05 & 06 \\
\hline Maternity hospital & 01 & - \\
\hline Baby nursery & 01 & 01 \\
\hline C. albicans & 10 & 06 \\
\hline C. tropicalis & 03 & 13 \\
\hline C. parapsilosis & 09 & 07 \\
\hline Others* & 03 & 03 \\
\hline Treatment with \\
antibiotics
\end{tabular}

Graph 1. Distribution of Candida species according to the hospital sector

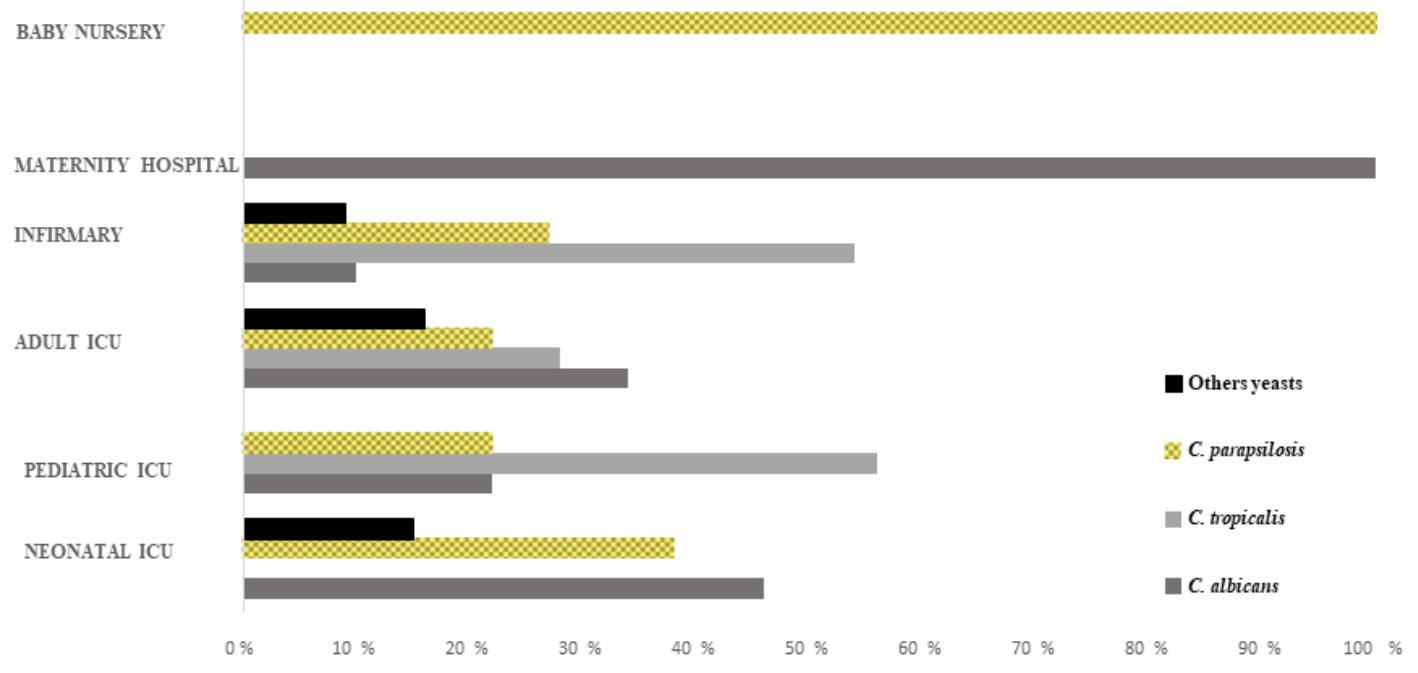


The predisposing factors for development of candidemia observed in our research corroborate the aforementioned studies (Rennert et al., 2000; Chang et al., 2008; Arendrup et al., 2011; Israel et al., 2019). Vibhor et al., (2014) have shown that the presence of bladder catheter, nasogastric tube, central catheter or dialysis, as well as prior administration of antibiotics were the main conditions associated with an increased risk. Most of the infected patients in our study used a bladder catheter or nasogastric tube and central catheter, and antibiotics were used in all who were sick before the development of candidemia. Berdal et al., (2014) also presented in their study antibiotic therapy prior to candidemia in $87.0 \%$ of patients, characterizing it as a risk factor for incidences of cases of infection by Candida spp.

Fungi have a high capacity to attach themselves to surfaces (devices, probes and catheters) forming a biofilm, and to protect themselves against antifungal agents and immune responses. These mechanisms may in fact explain the common association of infections by Candida spp. and the use of probes and catheters, procedures involving intubations, and tracheostomy. Something else to consider is prolonged hospital stays as an important risk factor as observed in our study.

In this research we analyzed the prognosis for patients with candidemia, and verified a high mortality rate $(51.8 \%)$, superior to those in hospitals in China (26.0 - 33.3\%) (Wu et al., 2011; Li et al., 2013), Europe (37.9\%) (Tortorano et al., 2004), but inferior to hospitals in Saudi Arabia, where the mortality rate is $72.0 \%$ (Al-Tawfiq, 2007). We also observed that $C$. tropicalis was the species associated with the highest mortality rate (46.4\%), corroborating the results encountered by Chung-Fang et al., (2013). The severity of the underlying medical conditions will have certainly influenced the mortality rate considerably among the population in study. It is also important to note that the hospital in this research is an institute of reference in the region for polytrauma patient care, who represent $16.6 \%$ of the deceased patients.

The main limitation of this study were the incomplete records, which made it impossible to faithfully collect data on some risk factors such as tobacco use, alcoholism, diabetes, etc.

Another point to consider is the lack of a protocol from the hospital to request microbiological tests for the cases that fit the criteria for inclusion in this study, and we can't therefore guarantee that all the cases that corresponded to our criteria had fungi cultures requested for them. Possible causes for this problem are the scarcity of resources for public hospitals in our country, as well as the unfamiliarity with this issue (candidemia) among nonspecialists.

In addition, the difficulty to put into practice consecrated procedures for the prevention of hospital infections, such as hand hygiene, to avoid transmission through the colonized hands of the team providing care to patients, as well as an insufficient number of health care professionals in critical areas, especially in the intensive care units, should also be considered.

The results of this research highlight the need to evaluate the impact of prolonged hospitalizations, indiscriminate use of broadspectrum antibacterials, comorbidities prior to hospitalization, patient age, and the presence of invasive devices in the incidence of nosocomial candidemia. Futhermore, epidemiological studies can also contribute to action plans for the improvement of hospital environments and therapy conducts.

Finally, this study points to the need to find ways to avoid invasive infections by Candida 
spp., which are rising rapidly in our region and throughout the world, and which also, represent a significant negative influence on the prognosis of the patients.

\section{Acknowledgments}

This study was supported in part by Santa Casa de Miseridórdia of Sobral, Ceará, Brazil (Edital DEPE 03/2015).

\section{References}

Ala-Houhala, M., Valkonen, M., Kolho, E., Friberg, N., and Veli-JukkaAnttila, V-J. 2019. Clinical and microbiological factors associated with mortality in candidemia in adult patients 2007-2016. Journal Infectious Diseases.51 (11-12): 824-830.

Allert, S., Förster, T.M., Svensson, C. M., Richardson, J. P., Pawlik, T., Betty H., Rudolphi, S., Juraschitz, M., Schaller, M., Blagojevic, M., Morschhäuser, J., Figge, M.T., Jacobsen, I. D., Naglik, J.R., Kasper, L., Mogavero, S., andHube, B. 2018. Candida albicans -Induced Epithelial Damage Mediates Translocation through Intestinal Barriers. Mbio.9 (3): 15-18.

Al-Rawahi G. N., and Roscoe D. L. 2013. Ten-year review of candidemia in a Canadian tertiary care centre: Predominance of non-albicans Candida species. Can J Infect Dis Med Microbiol. 24 (3): 123-128.

Al-Tawfiq J.A.2007. Distribution and epidemiology of Candida species causing fungemia at a Saudi Arabian hospital. Int J Infect Dis. 11 (3):239 - 244.

Arendrup, M.C., Bruun, B., Christensen, J.J., Fuursted, K., Johansen, H.K., Kjældgaard P, Knudsen JD, Kristensen L, Møller J, and Nielsen L. 2011. National surveillance of fungemia in Denmark (2004 to 2009). J Clin
Microbiol. 49 (1):325 - 334.

Bailly, S., Maubon, D., Fournier, P., Pelloux, H., Schwebel, C., Chapuis, C., Foroni, L., Cornet, M., and Timsit, J-F., 2016. Impact of antifungal prescription on relative distribution and susceptibility of Candida spp. - Trends over 10 years. Journal of Infection. 72 (1): 103111.

Bassetti, M., Righi E., and Costa A. 2006. Epidemiological trends in nosocomial candidemia in intensive care. BMC Infect Dis. 6 (21): 1-6.

Berdal J. E., Haagensen R., Ranheim T., and Bjørnholt J.V. 2014. Nosocomial Candidemia; Risk Factors and Prognosis Revisited; 11 Years Experience from a Norwegian Secondary Hospital. PLoSONE. 9 (7):1-6.

Chang M. R., Correia, F. P., Costa, L. C., Xavier, P. C. N., Palhares, D. B., Taira D. L., Paniago, A. M. M., Pontes, E. R. J.C., and Machado, V. E. 2008. Candida blood stream infection: data from a teaching hospital in MatoGrosso do Sul, Brazil. Rev Inst Med Trop. 50 (5): 265268.

Chatelon, J., Cortegiani, A., Hammad, E., Cassir, N., and Leone, M. 2019. Choosing the Right Antifungal Agent in ICU Patients. Advances in Therapy.36 (12): 3308-3320.

Chun-Fang M., Li F., Shi L.N., Hu, Y.A., Wang, Y., Huang, M., and Kong, Q.Q. 2013. Surveillance study of species distribution, antifungal susceptibility and mortality of nosocomial candidemia in a tertiary care 320 hospital in China. BMC Infect Dis. 13 (337): 1-9.

Cleveland, A.A., Harisson, L.H., Farley, M.M., Hollick, R., Stein, B., Chiller, T.M., Lockhart, S.R., and Park, B.J. 2015. Declining Incidence of Candidemia and the Shifting Epidemiology of Candida Resistance in Two US Metropolitan Areas, 2008- 
2013: Results from Population-Based Surveillance. PLoS One. 10 (3): 1-12.

Colombo A.L., Garnica M., Aranha C.L.F., Da Cunha, C.A., Bandeira, A.C., Borghi, D., Campos, T., Senna, A.L., Valias Didier, M.E., Dias, V.C., and Nucci, M. 2013. Candida glabrata: an emerging pathogen in Brazilian tertiary care hospitals. Med Mycol.51 (1): 38-44.

Dutta A., and Palazzi D. L. 2011. Candida non-albicans versus Candida albicans fungemia in the non-neonatal pediatric population. Pediatr Infect Dis J. 30 (8): 664-668.

Epelbaum O., and Chasan, R. 2017. Candidemia in the Intensive Care Unit. Clinics in Chest Medicine.38 (3): 493-509.

Ezenwa, B. N., Oladele, R.O., Akintan, P.E., Fajolu, I.B., Oshun, P.O., Oduyebo, O.O., and Ezeaka, V.C. 2017. Invasive candidiasis in a neonatal intensive care unit in Lagos, Nigeria. Nigerian Postgraduate Medical Journal. 24 (3): 150-154.

Hinrichsen, S. L., Falcão, E., Vilella, T. A. S., Colombo, A. L., Nucci, M., Moura, L., Rêgo, L., Lira, C. and Almeida, L. 2008. Candidemia in a tertiary hospital in north eastern Brazil. Soc. Bras Med Trop. 41 (4): 394-398.

Horn D. L., Neofytos D., and Anaissie E.J. 2009. Epidemiology and outcomes of candidemia in 2019 patients: Data from the prospective antifungal therapy alliance registry. Clin Infect Dis. 48 (12): 1695-1703.

Israel, S., Amit, S., Israel, A., Livneh, A., NirPaz, R., and Korem, M. 2019. The Epidemiology and Susceptibility of Candidemia in Jerusalem, Israel. Frontiers in Cellular and Infection Microbiology. 9 (1): 1- 7.

Labbé A. C., Pépin J., Patiño C., Castonguay S., Restieri C., and Laverdiere M.A. 2009. Single-centre 10-year experience with Candida bloods tream infections. Can J Infect Dis Med Microbiol. 20(2): 45-50.

Li, D., Zhang, W., Zheng, S., Ma, Z., Zhang, P., and Liu, Z. 2013. Surveillance study of candidemia in cancer patients in North China. Med Mycol. 51 (4): 378 - 384.

Lin, S., Chen, R., Zhu, S., Wang, H., Wang, L., Zou, J., Yan, J., Zhang, X., Farmakiotis, D., Tan, X., and Mylonakis, E. 2018. Candidemia in Adults at a Tertiary Hospital in China: Clinical Characteristics, Species Distribution, Resistance, and Outcomes. Mycopathologia. 183(4): 679-689.

Lortholary O., Desnos-Ollivier M., and Sitbon, K. 2011. French Mycosis Study Group: Recent exposure to caspofungin or fluconazole in fluences the epidemiology of candidemia: A prospective multicenter study involving 2,441 patients. Antimicrob Agents Chemother. 55(2): 532-538.

Motta, A.L., Almeida, G.M.D., Almeida Júnior, J.N.A, Burattini, M.N., and Rossi F. 2010. Candidemia epidemiology and susceptibility profile in the largest Brazilian teaching hospital complex. Braz J InfectDis.14 (5): 441-448.

Ortega M., Marco F., and Soriano A. 2011. Candida species blood stream infection: Epidemiology and outcome in a single institution from 1991 to 2008. J Hosp Infect. 77(2): 350- 361.

Pinto- MagalhãesS., Martins, A., Lacerda, S., Rita, F., Leão, B.P., Dolores, P., SilvaPinto, A., and Santos, L. 2019. Candidemia in a Portuguese tertiary care hospital: Analysis of a 2-year period. Journal de Mycologie Médicale. 29(4): 320-324.

Rennert, G., Rennert, H.S., Pitlik, S., Finkelstein, R., and Kitzes-Cohen R. 2000. Epidemiology of candidemia - a nationwide survey in Israel. Infection. 28(1): $26-29$. 
Roilides E. 2011. Invasive candidias is in neonates and children. Early Hum Rev., 87 (Suppl 1): 75-76.

Shetty S.S., Harrison L.H, Hajjeh R.A., Taylor T., Mirza S.A., Schmidt A.B, Sanza L.T, Shutt K.A, and Fridkin S.K. 2005. Determining risk factors for candidemia among new born infants from population - based surveillance: Baltimore, Maryland, 1998-2000. Pediatr Infect Dis J. 24 (7): $601-604$.

St-Germain, G., Laverdière, M., and Pelletier, R. 2008. Epidemiology and antifungal susceptibility of blood stream Candida isolates in Quebec: Reporton 453 cases between 2003 and 2005. Can J Infect Dis Med Microbiol. 19(1): 55-62.

Tortorano A., Peman, J., Bernhard, H., Klingspor, L., Kibbler, C. C., Faure, O., Biraghi, E., Canton, E., Zimmermann, K., Seaton, S., and Grillot, R. 2004. Epidemiology of candidaemiain Europe: results of 28-month European Confederation of Medical Mycology (ECMM) hospital based surveillance study. Eur J Clin Microbiol Infect Dis.
23(4): $317-322$.

Vibhor T.A.K., Purva M., Varghese, P., Gunjiyal, J., Xess I., and Misra, M.C. 2014. Epidemiological Profile of Candidemia at an Indian Trauma Care Center Mahesh C Misra. J Lab Physicians. 6 (2): 96 -101.

Wille, M.P., Guimarães, T., Furtado, G.H., and Colombo, A.L. 2013. Historical trends in the epidemiology of candidaemia: analysis of an 11-year period in a tertiary care hospital in Brazil. Mem Inst Oswaldo Cruz. 108 (3): 288-292.

Wu J.Q., Zhu L. P., Ou X. T., Xu, B., Hu, X. P., Wang, X., andWeng X. H. 2011. Epidemiology and factors for nonCandida albicans candidemia in nonneutropenic patientsat a Chinese teaching hospital. Med Mycol. 49 (5): 552 - 555.

Yesilkaya A., Azap Ö., Aydin M., and Akçil O.K.M., 2017. Epidemiology, species distribution, clinical characteristics and mortality of candidaemia in a tertiary care university hospital in Turkey, 20072014. Mycoses. 60 (7):433-439.

\section{How to cite this article:}

Vilson Sovio Oliveira de Macedo, Sebastião Carlos de Sousa Oliveira, Maria Rosineida Paiva Rodrigues, Camila Gomes Virginio Coelho and Francisco Cesar Barroso Barbosa. 2019. Prevalence of Candida in blood Cultures from in patients at a Teaching Hospital in Brazil. Int.J.Curr.Microbiol.App.Sci. 8(12): 2063-2073. doi: https://doi.org/10.20546/ijcmas.2019.812.244 